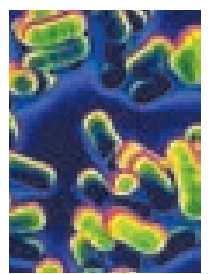

Unité de Recherche Yersinia, Centre National de Référence et Centre Collaborateur de l'OMS pour les Yersinia, Institut Pasteur, 28, rue du Docteur Roux, 75724 Paris Cedex 15, France. carniel2@pasteur.fr

\section{Qu'est-ce que la peste?}

La peste est une infection bactérienne aiguë causée par le bacille Yersinia pestis. II s'agit avant tout d'une zoonose, touchant principalement des rongeurs, sauvages ou péridomestiques (rats). La maladie est transmise de rongeur à rongeur par piqûre d'une puce vectrice (Figure 1). L'infection humaine ne représente qu'un épiphénomène de ces enzooties. Lorsqu'un être humain est piqué par une puce de rongeur infecté, il développe un syndrome infectieux sévère accompagné d'une adénopathie volumineuse et exquisément douloureuse dans le territoire de drainage du point de piqûre de la puce. Cette adénopathie est appelée bubon. Une peste bubonique non traitée est fatale, généralement en moins d'une semaine, dans $40 \%$ à $70 \%$ des cas. Une complication possible de la peste bubonique est le développement d'une pneumopathie pesteuse, ou peste pulmonaire. Cette maladie d'évolution foudroyante (moins de 3 jours), constamment mortelle en absence d'un traitement précoce, est directement contagieuse d'homme à homme par le biais des gouttelettes respiratoires émises lors de la toux (Figure 1).

\section{Situation actuelle}

\section{Répartition géographique mondiale}

Quoique la peste ne soit plus de nos jours le fléau qu'elle a été par le passé, grâce en particulier à la découverte de l'agent causal, du cycle de transmission, et surtout grâce à I'utilisation de traitements antibiotiques efficaces, elle est loin d'avoir été éradiquée. Près de 44000 cas humains ont été déclarés à l'OMS par 26 pays au cours des vingt dernières années (Tableau I) [1, 2], et le nombre réel de cas est probablement beaucoup plus élevé du fait des difficultés de diagnostic ou de la notification insuffisante par certains pays. Les plus gros contingents rapportés à l'OMS sont en Afrique orientale, avec en particulier des foyers très actifs à Madagascar, en République Démocratique du Congo et en Tanzanie. Viennent ensuite l'Asie du Sud-Est (Vietnam, Chine), l'Asie centrale (Mongolie, Kazakhstan, Inde), et les deux sous-continents américains. Aux Etats-Unis, des foyers actifs de peste sylvatique occupent le tiers ouest du pays et sont responsables chaque année de cas humains.

\section{Extension et réémergence des foyers}

Le nombre de cas humains de peste rapportés à l'OMS a augmenté significativement depuis le début des années 1990. Parmi les épidémies les plus récentes et d'une certaine ampleur, citons le cas de celle qui sévit dans la République Démocratique du Congo, où trois épidémies meurtrières de peste pulmonaire ont eu lieu en 2005 [3] et 2006, dont deux dans des régions minières situées en dehors des foyers connus de peste de ce pays. Une extension des zones endémiques, et même la résurgence de foyers que l'on croyait éteints, sont également observées depuis une quinzaine d'années (Figure 2). Cela a été le cas au niveau de la zone côtière de Mahajanga (Madagascar) en 1991, après 60 ans de silence. En Inde, la peste semblait avoir disparu depuis 1964 mais est réapparue en 1994, sous la forme d'une épidémie de peste pulmonaire qui a paralysé la vie économique du pays pendant plusieurs semaines [4]. À partir du Mozambique, où elle est réapparue en 1994 après 15 ans d'absence, la maladie s'est propagée au Zimbabwe et au Malawi. En Zambie, une épidémie de peste bubonique s'est déclarée fin 1996 alors qu'aucun cas humain n'y avait été signalé depuis plus de 30 ans. Des cas ont été rapportés en 1997 en Jordanie, plus de 80 ans après les derniers cas recensés dans ce pays. Plus récemment, 


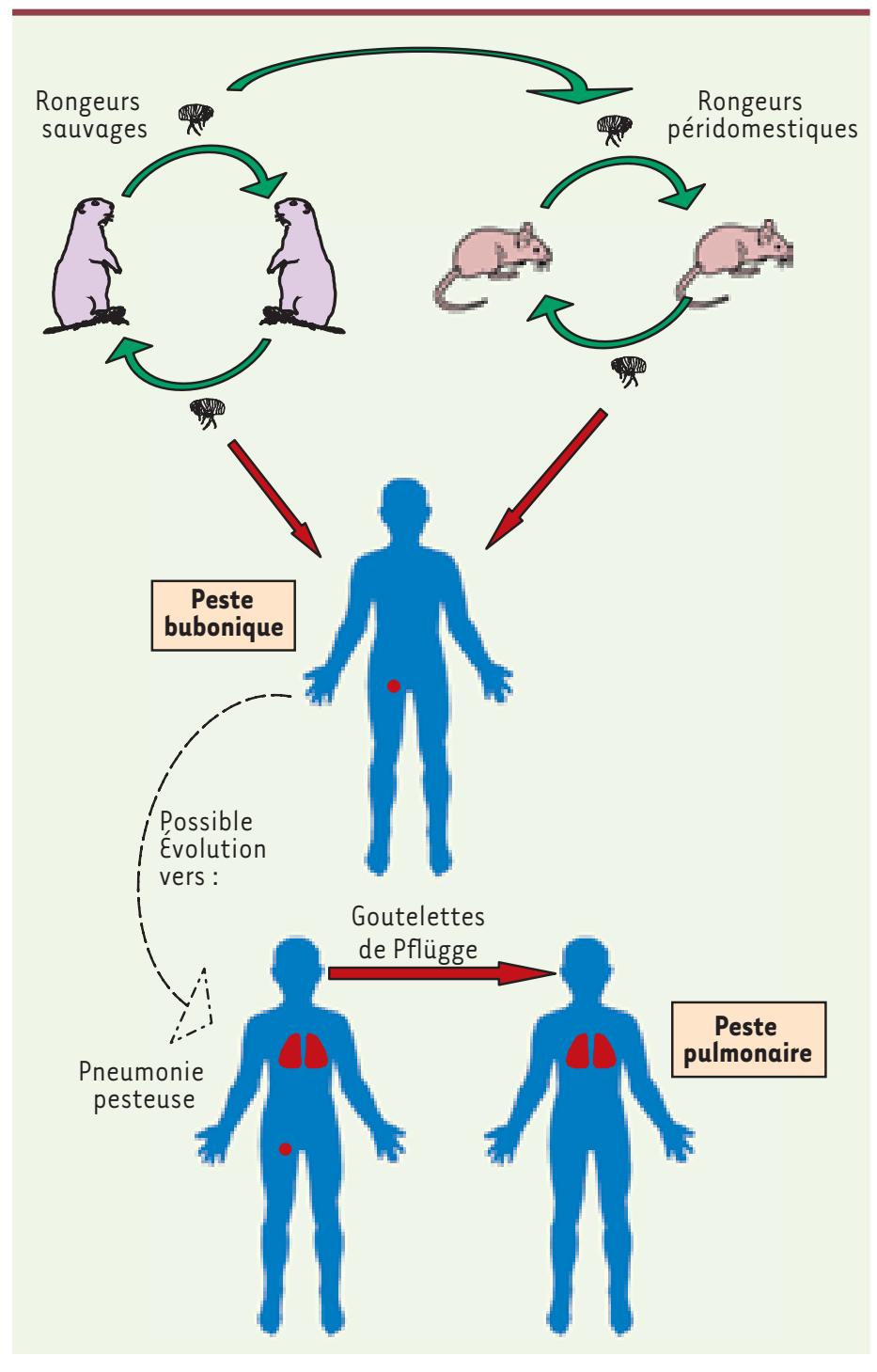

Figure 1. La peste, zoonose touchant principalement des rongeurs, sauvages ou péridomestiques (rats), transmise de rongeur à rongeur par piqûre d'une puce vectrice.

en 2003, la peste est revenue dans la région d'Oran en Algérie [5] alors qu'aucun cas n'avait été signalé dans ce pays depuis la célèbre Peste d'Albert Camus [6].

\section{Diagnostic}

\section{Diagnostic bactériologique classique}

L'isolement du bacille pesteux et sa caractérisation à partir d'échantillons cliniques (ponctions de bubon, hémocultures, expectorations ou prélèvements postmortem) apportent un diagnostic de certitude mais tardif (environ une semaine).

\section{Diagnostic rapide}

La précocité du diagnostic étant cruciale pour la prise en charge des patients et la mise en oeuvre de mesures prophylactiques pour l'entourage, un diagnostic rapide est nécessaire. Des tests diagnostiques par PCR ont été développés depuis quelques années, mais leur utilisation dans les conditions de terrain n'est pas réalisable. Un test d'immunodétection sur bandelettes a récemment été mis au point [7]. Ce test est rapide (moins de 10 minutes), sensible, spécifique, et peut être effectué directement au lit du malade par les agents de santé, ce qui représente un progrès majeur dans le diagnostic précoce de la maladie en zones d'endémies pesteuses.

\section{Traitement et résistance aux antibiotiques}

\section{Traitements curatifs et prophylactiques}

Le traitement curatif de la peste repose sur l'administration précoce d'antibiotiques. Ceux qui sont recommandés par l'OMS sont la streptomycine, les cyclines et le chloramphénicol, utilisés généralement en monothérapie. Plus récemment, les fluoroquinolones et la gentamycine sont apparues comme de nouvelles options thérapeutiques. Les formes évoluées qui s'accompagnent de choc septique et/ou d'insuffisance respiratoire requièrent un traitement symptomatique associé en unité de soins intensifs. L'antibioprophylaxie des sujets contacts repose sur les sulfamides ou la tétracycline. Il n'y a pas à ce jour de vaccin efficace et bien toléré contre la peste.

Figure 2. Ré-emergence récente de la peste dans des foyers que l'on croyait éteints depuis plusieurs décennies. 


\section{Apparition de résistances aux antibiotiques}

Le bacille pesteux est habituellement sensible aux antibiotiques classiquement utilisés pour le traitement de la peste. Cependant, on sait depuis peu que le risque d'apparition de chimiorésistances étendues chez $Y$. pestis existe. En 1995, deux souches portant des plasmides de résistance à des antibiotiques de première ligne pour le traitement de la peste ont été isolées chez des patients atteints de peste bubonique. Un de ces plasmides conférait un taux de résistance important à la streptomycine - antibiotique de choix pour le traitement de la peste dans de nombreux pays -, et l'autre une résistance à huit antibiotiques, dont les quatre recommandés par l'OMS comme traitements curatif et prophylactique) [8]. Dans les deux cas, le plasmide était capable de se transférer avec une fréquence élevée d'une souche de $y$. pestis à une autre. La démonstration ultérieure que l'acquisition par $y$. pestis de cette résistance aux antibiotiques avait lieu dans le tube digestif de la puce [9] et que le plasmide de multirésistance était présent chez des entérobactéries largement répandues dans les produits alimentaires [10] fait redouter l'émergence et l'expansion de souches de $y$. pestis multirésistantes, ce qui constituerait une menace majeure en santé publique.

\begin{tabular}{|c|c|c|}
\hline Afrique & $\begin{array}{l}\text { Nombre } \\
\text { de cas }\end{array}$ & $\begin{array}{l}\text { Nombre } \\
\text { de décès }\end{array}$ \\
\hline Algérie & 11 & 1 \\
\hline Bostwana & 173 & 12 \\
\hline Rép. Démocr. Congo & 5778 & 862 \\
\hline Kenya & 44 & 8 \\
\hline Lybie & 8 & 0 \\
\hline Madagascar & 13417 & 1207 \\
\hline Malawi & 907 & 16 \\
\hline Mozambique & 2387 & 28 \\
\hline Namibie & 2189 & 81 \\
\hline Ouganda & 1161 & 157 \\
\hline Rép. Unie de Tanzanie & 7344 & 522 \\
\hline Zambie & 1170 & 29 \\
\hline Zimbabwe & 419 & 36 \\
\hline Total Afrique & 35008 & 2959 \\
\hline \multicolumn{3}{|l|}{ Amériques } \\
\hline Bolivie & 147 & 24 \\
\hline Brésil & 332 & 6 \\
\hline Équateur & 24 & 16 \\
\hline Pérou & 1878 & 105 \\
\hline États-Unis d'Amérique & 186 & 20 \\
\hline Total Amériques & 2567 & 171 \\
\hline \multicolumn{3}{|l|}{ Asie } \\
\hline Chine & 546 & 63 \\
\hline Inde & 892 & 58 \\
\hline Indonésie & 6 & 0 \\
\hline Kazakhstan & 24 & 7 \\
\hline Rép. Democr. Pop. Du Laos & 10 & 0 \\
\hline Mongolie & 116 & 36 \\
\hline Myanmar & 825 & 6 \\
\hline Viet Nam & 3862 & 202 \\
\hline Total Asie & 6281 & 372 \\
\hline TOTAL MONDE & 43856 & 3502 \\
\hline
\end{tabular}

Tableau I. Nombre de cas de peste déclarés à l'OMS au cours des deux dernières décennies (1984-2003).

\section{Conclusion : la peste représente-t-elle toujours une menace?}

La peste ne se présente plus aujourd'hui sous la forme des épidémies massives et dévastatrices qui ont marqué l'histoire et la mémoire collective des hommes. Cependant, cette maladie pose encore au monde actuel d'importants défis.

Certains sont liés aux caractéristiques mêmes de la maladie: gravité, contagiosité (pour la forme pulmonaire), rapidité de dissémination, qui imposent une bonne réactivité sur le terrain devant un début d'épidémie. À ces caractéristiques s'ajoute l'effet de panique qui peut, entre autres effets pervers, favoriser la dissémination. La disponibilité de nos jours de moyens de transport rapides permet à un malade en phase d'incubation d'importer la maladie dans des régions très distantes de la source d'infection.

Un autre défi est constitué par la réapparition récente de la peste dans des foyers restés silencieux pendant plusieurs décennies, indiquant que l'éradication d'un foyer de peste établi ne peut jamais être tenue pour acquise. L'extension des foyers existants s'explique au moins en partie par des changements anthropogéniques du paysage (fragmentation des forêts) et les déplacements humains qui favorisent considérablement les contacts entre le réservoir des animaux sauvages et les rongeurs péridomestiques, ainsi qu'entre les populations des zones endémiques et celles des zones non encore affectées. De plus, les modifications climatiques observées actuellement (printemps plus chauds, étés plus humides) offrent des conditions favorables à l'apparition d'épizooties de peste [1] et donc au risque de transmission à l'homme.

Enfin, l'analyse des foyers de peste actuels indique que cette maladie est ubiquitaire géographiquement (foyers dispersés dans le monde) et dans le règne animal (nombreuses espèces hôtes et puces vectrices), ce qui atteste d'une capacité remarquable de la bactérie à s'adapter à des conditions écologiques variées et rend utopique une 
éradication totale de la maladie. À cela s'ajoute une menace potentielle majeure liée à la capacité maintenant démontrée du bacille à acquérir et transmettre des plasmides de résistance aux antibiotiques dans des conditions naturelles.

Loin d'être uniquement une maladie du passé ou un problème limité à des zones géographiques restreintes, la peste représente donc encore de nos jours un danger potentiel à l'échelle de la planète. Le maintien, ou même le renforcement, d'une surveillance de terrain active et les recherches visant à améliorer nos connaissances sur les conditions de circulation du bacille et à identifier de nouvelles cibles thérapeutiques restent plus que jamais nécessaires. $\diamond$

Should we still fear the plague today?

\section{RÉFÉRENCES}

1. WHO. Human plague in 1996. Wkly Epidemiol Rec 1998 ; $47: 366-9$.

2. WHO. Human plague in 2002 and 2003. Wkly Epidemiol Rec 2004 ; $79: 301-8$.

3. Bertherat $\varepsilon$, Lamine KM, Formenty $P$, et al. Major pulmonary plague outbreak in a mining camp in the Democratic Republic of Congo: brutal awakening of an old scourge. Med Trop $2005 ; 65$ : 511-4.

4. Ramalingaswami V. Plague in India. Nat Med $1995 ; 1$ : 1237-9.

5. Bertherat $\varepsilon$, Bekhoucha $S$, Chougrani $S$, et al. Plague reappearance in Algeria after 50 years, 2003. Emerg Infect Dis 2007; 13 : 1459-62.

6. Camus A. La Peste. Paris: Gallimard, 1947.

7. Chanteau S, Rahalison L, Ralafiarisoa L, et al. Development and testing of a rapid diagnostic test for bubonic and pneumonic plague. Lancet 2003; 361:211-6.

8. Galimand M, Carniel $\varepsilon$, Courvalin P. Resistance of Yersinia pestis to antimicrobial agents. Antimicrob Agents Chemother 2006 ; 50 : 3233-6.

9. Hinnebusch BJ, Rosso ML, Schwan TG, Carniel $\varepsilon$. High-frequency conjugative transfer of antibiotic resistance genes to Yersinia pestis in the flea midgut. Mol Microbiol $2002 ; 46: 349-54$.

10. Welch TJ, Fricke WF, McDermott PF, et al. Multiple antimicrobial resistance in plague: an emerging public health risk. PLoS One 2007 ; 2 : e309.

11. Davis $S$, Begon M, De Bruyn L, et al. Predictive thresholds for plague in Kazakhstan. Science 2004 ; 304 : 736-8.

$\varepsilon$. Carniel

\section{Collection SCIENCE ET BIOMÉDECINE}

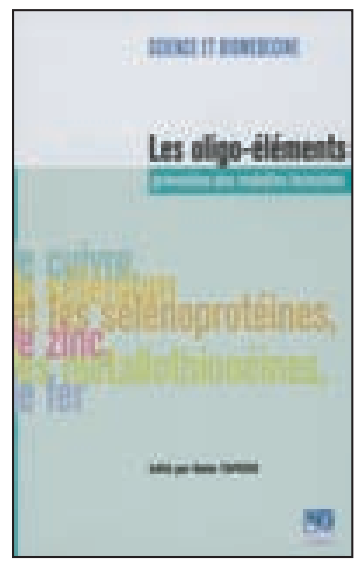

ISBN : 2-84254-107-3 64 pages

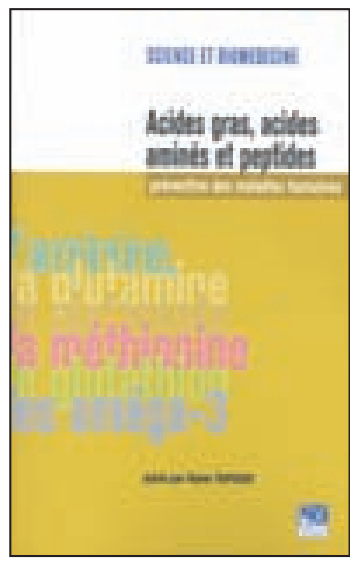

ISBN : 2-84254-108-1 80 pages

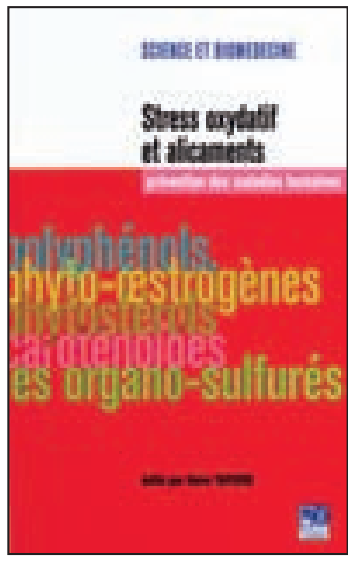

ISBN : 2-84254-111-1 86 pages

\section{Bon de commande}

À retourner à EDK, 2, rue Troyon - 92316 Sèvres Cedex

Tél. : 0155641393 - Fax : 0155641394 - E-mail : edk@edk.fr

NOM :

Prénom :

Adresse :

Code postal :

Ville :

Pays :

Fonction :

Je souhaite recevoir l'ouvrage Les oligo-éléments : $10 €+3 €$ de port $=\mathbf{1 3} €$ TTC

Je souhaite recevoir l'ouvrage Acides gras, acides aminés et peptides : $12 €+3 €$ de port $=\mathbf{1 5} €$ TTC

Je souhaite recevoir l'ouvrage Stress oxydatif et alicaments : $14 €+3 €$ de port $=17 €$ TTC

en .. exemplaire, soit un total de $€$

$\square$ Par chèque, à l'ordre de $\mathbf{E} \mathbf{D}$ K

Par carte bancaire : $\square$ Visa $\square$ Eurocard/Mastercard

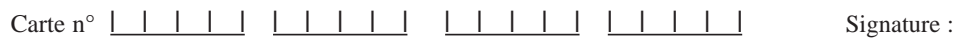

Date d'expiration: $\quad \underline{1 \quad 1 \quad 1 \quad 1}$

$\mathrm{N}^{\circ}$ de contrôle au dos de la carte : 